\title{
Article
}

\section{Positive solutions for nonlinear Caputo-Hadamard fractional differential equations with integral boundary conditions}

\author{
Abdelouaheb Ardjouni ${ }^{1,2, *}$ and Ahcene Djoudi ${ }^{2}$ \\ 1 Department of Mathematics and Informatics, University of Souk Ahras, P.O. Box 1553, Souk Ahras, 41000, Algeria. \\ 2 Applied Mathematics Lab, Faculty of Sciences, Department of Mathematics, University of Annaba, P.O. Box 12, \\ Annaba 23000, Algeria.; adjoudi@yahoo.com(A.D) \\ * Correspondence: abd_ardjouni@yahoo.fr
}

Received: 02 April 2019; Accepted: 26 April 2019; Published: 28 May 2019.

\begin{abstract}
We study the existence and uniqueness of positive solutions of the nonlinear fractional differential equation with integral boundary conditions $\mathfrak{D}_{1}^{\alpha} x(t)=f(t, x(t)), \quad 1<t \leq e, x(1)=\lambda \int_{1}^{e} x(s) d s+d$, where $\mathfrak{D}_{1}^{\alpha}$ is the Caputo-Hadamard fractional derivative of order $0<\alpha \leq 1$. In the process we convert the given fractional differential equation into an equivalent integral equation. Then we construct an appropriate mapping and employ the Schauder fixed point theorem and the method of upper and lower solutions to show the existence of a positive solution of this equation. We also use the Banach fixed point theorem to show the existence of a unique positive solution. Finally, an example is given to illustrate our results.
\end{abstract}

Keywords: Fixed points, positive solutions, upper and lower solutions, fractional differential equations, existence, uniqueness.

MSC: 34A08, 34A12, 34B15, 34B18.

\section{Introduction}

F ractional differential equations with and without delay arise from a variety of applications including in various fields of science and engineering such as applied sciences, practical problems concerning mechanics, the engineering technique fields, economy, control systems, physics, chemistry, biology, medicine, atomic energy, information theory, harmonic oscillator, nonlinear oscillations, conservative systems, stability and instability of geodesic on Riemannian manifolds, dynamics in Hamiltonian systems, etc. In particular, problems concerning qualitative analysis of linear and nonlinear fractional differential equations with and without delay have received the attention of many authors, see [1-15] and the references therein.

Zhang in [16] investigated the existence and uniqueness of positive solutions for the nonlinear fractional differential equation

$$
\left\{\begin{array}{l}
D^{\alpha} x(t)=f(t, x(t)), 0<t \leq 1, \\
x(0)=0,
\end{array}\right.
$$

where $D^{\alpha}$ is the standard Riemann Liouville fractional derivative of order $0<\alpha<1$ and $f:[0,1] \times[0, \infty) \rightarrow$ $[0, \infty)$ is a given continuous function. By using the method of the upper and lower solution and cone fixed-point theorem, the author obtained the existence and uniqueness of a positive solution.

The nonlinear fractional differential equation

$$
\left\{\begin{array}{l}
{ }^{C} D^{\alpha} x(t)=f(t, x(t))+{ }^{C} D^{\alpha-1} g(t, x(t)), 0<t \leq T, \\
x(0)=\theta_{1}>0, x^{\prime}(0)=\theta_{2}>0,
\end{array}\right.
$$

has been investigated in [5], where ${ }^{C} D^{\alpha}$ is the standard Caputo's fractional derivative of order $1<\alpha \leq 2$, $g, f:[0, T] \times[0, \infty) \rightarrow[0, \infty)$ are given continuous functions, $g$ is non-decreasing on $x$ and $\theta_{2} \geq g\left(0, \theta_{1}\right)$. By 
employing the method of the upper and lower solutions and Schauder and Banach fixed point theorems, the authors obtained positivity results.

In [2], Abdo, Wahash and Panchat discussed the existence and uniqueness of positive solutions of the following nonlinear fractional differential equation with integral boundary conditions

$$
\left\{\begin{array}{l}
{ }^{c} D^{\alpha} x(t)=f(t, x(t)), 0<t \leq 1 \\
x(0)=\lambda \int_{0}^{1} x(s) d s+d
\end{array}\right.
$$

where $0<\alpha \leq 1, \lambda \geq 0, d>0$ and $f:[0,1] \times[0, \infty) \rightarrow[0, \infty)$ is a given continuous function. By using the method of the upper and lower solutions and Schauder and Banach fixed point theorems, the existence and uniqueness of solutions has been established.

Ahmad and Ntouyas in [4] studied the existence and uniqueness of solutions to the following boundary value problem

$$
\left\{\begin{array}{l}
\mathfrak{D}_{1}^{\alpha}\left(\mathfrak{D}_{1}^{\beta} u(t)-g\left(t, u_{t}\right)\right)=f\left(t, u_{t}\right), t \in[1, b], \\
u(t)=\phi(t), t \in[1-r, 1], \\
\mathfrak{D}_{1}^{\beta} u(1)=\eta \in \mathbb{R},
\end{array}\right.
$$

where $\mathfrak{D}_{1}^{\alpha}$ and $\mathfrak{D}_{1}^{\beta}$ are the Caputo-Hadamard fractional derivatives, $0<\alpha, \beta<1$. By employing the fixed point theorems, the authors obtained existence and uniqueness results.

In this paper, we are interested in the analysis of qualitative theory of the problems of the positive solutions to fractional differential equations with integral boundary conditions. Inspired and motivated by the works mentioned above and the papers [1-15] and the references therein, we concentrate on the positivity of the solutions for the nonlinear fractional differential equation with integral boundary conditions

$$
\left\{\begin{array}{l}
\mathfrak{D}_{1}^{\alpha} x(t)=f(t, x(t)), 1<t \leq e, \\
x(1)=\lambda \int_{1}^{e} x(s) d s+d,
\end{array}\right.
$$

where $\mathfrak{D}_{1}^{\alpha}$ is the Caputo-Hadamard fractional derivative of order $0<\alpha \leq 1, \lambda \geq 0, d>0, f:[1, e] \times[0, \infty) \rightarrow$ $[0, \infty)$ is a given continuous function. To show the existence and uniqueness of positive solutions, we transform (1) into an integral equation and then by the method of upper and lower solutions and use the Schauder and Banach fixed point theorems.

This paper is organized as follows. In Section 2, we introduce some notations and lemmas, and state some preliminaries results needed in later sections. Also, we present the inversion of (1) and the Banach and Schauder fixed point theorems. For details on the Banach and Schauder theorems we refer the reader to [15]. In Sections 3 and ??, we give and prove our main results on positivity and we provide an example to illustrate our results.

\section{Preliminaries}

Let $X=C([1, e])$ be the Banach space of all real-valued continuous functions defined on the compact interval $[1, e]$, endowed with the maximum norm. Define the cone

$$
\mathcal{E}=\{x \in X: x(t) \geq 0, \forall t \in[1, e]\} .
$$

We introduce some necessary definitions, lemmas and theorems which will be used in this paper. For more details, see [10,14].

Definition 1 ([10]). The Hadamard fractional integral of order $\alpha>0$ for a continuous function $x:[1,+\infty) \rightarrow \mathbb{R}$ is defined as

$$
\mathfrak{I}_{1}^{\alpha} x(t)=\frac{1}{\Gamma(\alpha)} \int_{1}^{t}\left(\log \frac{t}{s}\right)^{\alpha-1} x(s) \frac{d s}{s}, \alpha>0 .
$$


Definition 2 ([10]). The Caputo-Hadamard fractional derivative of order $\alpha>0$ for a continuous function $x:[1,+\infty) \rightarrow \mathbb{R}$ is defined as

$$
\mathfrak{D}_{1}^{\alpha} x(t)=\frac{1}{\Gamma(n-\alpha)} \int_{1}^{t}\left(\log \frac{t}{s}\right)^{n-\alpha-1} \delta^{n}(x)(s) \frac{d s}{s}, n-1<\alpha<n,
$$

where $\delta^{n}=\left(t \frac{d}{d t}\right)^{n}, n \in \mathbb{N}$.

Lemma 3 ([10]). Let $n-1<\alpha \leq n, n \in \mathbb{N}$ and $x \in C^{n}([1, T])$. Then

$$
\left(\mathfrak{I}_{1}^{\alpha} \mathfrak{D}_{1}^{\alpha} x\right)(t)=x(t)-\sum_{k=0}^{n-1} \frac{x^{(k)}(1)}{\Gamma(k+1)}(\log t)^{k} .
$$

Lemma 4 ([10]). For all $\mu>0$ and $v>-1$,

$$
\frac{1}{\Gamma(\mu)} \int_{1}^{t}\left(\log \frac{t}{s}\right)^{\mu-1}(\log s)^{v} \frac{d s}{s}=\frac{\Gamma(v+1)}{\Gamma(\mu+v+1)}(\log t)^{\mu+v} .
$$

The following lemma is fundamental to our results.

Lemma 5. Let $x \in C([1, e]), x^{\prime}$ exists, then $x$ is a solution of (1) if and only if

$$
x(t)=\frac{1}{\Gamma(\alpha)} \int_{1}^{t}\left(\log \frac{t}{s}\right)^{\alpha-1} f(s, x(s)) \frac{d s}{s}+\lambda \int_{1}^{e} x(s) d s+d, \quad 1 \leq t \leq e .
$$

Proof. Suppose $x$ satisfies (1), then applying $\mathfrak{I}_{1}^{\alpha}$ to both sides of (1), we have

$$
\mathfrak{I}_{1}^{\alpha} \mathfrak{D}_{1}^{\alpha} x(t)=\mathfrak{I}_{1}^{\alpha} f(t, x(t)) .
$$

In view of Lemma 3 and the integral boundary condition, we get

$$
x(t)=\frac{1}{\Gamma(\alpha)} \int_{1}^{t}\left(\log \frac{t}{s}\right)^{\alpha-1} f(s, x(s)) \frac{d s}{s}+\lambda \int_{1}^{e} x(s) d s+d, \quad 1 \leq t \leq e .
$$

Conversely, suppose $x$ satisfies (2), then applying $\mathfrak{D}_{1}^{\alpha}$ to both sides of (2), we obtain

$$
\begin{aligned}
\mathfrak{D}_{1}^{\alpha} x(t) & =\mathfrak{D}_{1}^{\alpha}\left(\frac{1}{\Gamma(\alpha)} \int_{1}^{t}\left(\log \frac{t}{s}\right)^{\alpha-1} f(s, x(s)) \frac{d s}{s}+\lambda \int_{1}^{e} x(s) d s+d\right) \\
& =\mathfrak{D}_{1}^{\alpha} \mathfrak{J}_{1}^{\alpha} f(t, x(t))+\mathfrak{D}_{1}^{\alpha}\left(\lambda \int_{1}^{e} x(s) d s+d\right) \\
& =f(t, x(t)) .
\end{aligned}
$$

Moreover, the integral boundary condition $x(1)=\lambda \int_{1}^{e} x(s) d s+d$ holds.

Lastly in this section, we state the fixed point theorems which enable us to prove the existence and uniqueness of a positive solution of (1).

Definition 6. Let $(X,\|\|$.$) be a Banach space and \mathcal{A}: X \rightarrow X$. The operator $\mathcal{A}$ is a contraction operator if there is an $\lambda \in(0,1)$ such that $x, y \in X$ imply

$$
\|\mathcal{A} x-\mathcal{A} y\| \leq \lambda\|x-y\|
$$

Theorem 7 (Banach [15]). Let $\mathcal{K}$ be a nonempty closed convex subset of a Banach space $X$ and $\mathcal{A}: \mathcal{K} \rightarrow \mathcal{K}$ be a contraction operator. Then there is a unique $x \in \mathcal{K}$ with $\mathcal{A} x=x$. 
Theorem 8 (Schauder [15]). Let $\mathcal{K}$ be a nonempty bounded, closed and convex subset of a Banach space $X$ and $\mathcal{A}$ : $\mathcal{K} \rightarrow \mathcal{K}$ be a completely continuous operator. Then $\mathcal{A}$ has a fixed point in $\mathcal{K}$.

\section{Existence of positive solutions}

In this section, we consider the results of existence problems for many cases of (1). We express (2) as

$$
x(t)=(\mathcal{A} x)(t)
$$

where the operators $\mathcal{A}: \mathcal{E} \rightarrow X$ is defined by

$$
(\mathcal{A} x)(t)=\frac{1}{\Gamma(\alpha)} \int_{1}^{t}\left(\log \frac{t}{s}\right)^{\alpha-1} f(s, x(s)) \frac{d s}{s}+\lambda \int_{1}^{e} x(s) d s+d .
$$

We need the following lemmas to establish our results.

Lemma 9. Assume that $f:[1, e] \times[0, \infty) \rightarrow[0, \infty)$ is continuous. Then, the operator $\mathcal{A}: \mathcal{E} \rightarrow \mathcal{E}$ is completely continuous.

Proof. By taking into account that $f$ is continuous nonnegative function, we get that $\mathcal{A}: \mathcal{E} \rightarrow \mathcal{E}$ is continuous. The function $f:[1, e] \times B_{\eta} \rightarrow[0, \infty)$ is bounded, then there exists $\rho>0$ such that

$$
0 \leq f(t, x(t)) \leq \rho,
$$

where $B_{\eta}=\{x \in \mathcal{E},\|x\| \leq \eta\}$. We obtain

$$
\begin{aligned}
|(\mathcal{A} x)(t)| & =\left|\frac{1}{\Gamma(\alpha)} \int_{1}^{t}\left(\log \frac{t}{s}\right)^{\alpha-1} f(s, x(s)) \frac{d s}{s}+\lambda \int_{1}^{e} x(s) d s+d\right| \\
& \leq \frac{1}{\Gamma(\alpha)} \int_{1}^{t}\left(\log \frac{t}{s}\right)^{\alpha-1}|f(s, x(s))| \frac{d s}{s}+\lambda \int_{1}^{e}|x(s)| d s+d \\
& \leq \frac{\rho}{\Gamma(\alpha)} \int_{1}^{t}\left(\log \frac{t}{s}\right)^{\alpha-1} \frac{d s}{s}+\lambda(e-1) \eta+d \\
& \leq \frac{\rho(\log t)^{\alpha}}{\Gamma(\alpha+1)}+\lambda(e-1) \eta+d .
\end{aligned}
$$

Thus,

$$
\|\mathcal{A} x\| \leq \frac{\rho}{\Gamma(\alpha+1)}+\lambda(e-1) \eta+d .
$$

Hence, $\mathcal{A}\left(B_{\eta}\right)$ is uniformly bounded.

Now, we will prove that $\mathcal{A}\left(B_{\eta}\right)$ is equicontinuous. Let $x \in B_{\eta}$, then for any $t_{1}, t_{2} \in[1, e], t_{2}>t_{1}$, we have

$$
\begin{aligned}
\left|(\mathcal{A} x)\left(t_{2}\right)-(\mathcal{A} x)\left(t_{1}\right)\right|= & \left|\frac{1}{\Gamma(\alpha)} \int_{1}^{t_{2}}\left(\log \frac{t_{2}}{s}\right)^{\alpha-1} f(s, x(s)) \frac{d s}{s}-\frac{1}{\Gamma(\alpha)} \int_{1}^{t_{1}}\left(\log \frac{t_{1}}{s}\right)^{\alpha-1} f(s, x(s)) \frac{d s}{s}\right| \\
\leq & \frac{1}{\Gamma(\alpha)} \int_{1}^{t_{1}}\left|\left(\log \frac{t_{2}}{s}\right)^{\alpha-1}-\left(\log \frac{t_{1}}{s}\right)^{\alpha-1}\right||f(s, x(s))| \frac{d s}{s} \\
& +\frac{1}{\Gamma(\alpha)} \int_{t_{1}}^{t_{2}}\left(\log \frac{t_{2}}{s}\right)^{\alpha-1}|f(s, x(s))| \frac{d s}{s} \\
\leq & \frac{\rho}{\Gamma(\alpha)} \int_{1}^{t_{1}}\left(\log \frac{t_{1}}{s}\right)^{\alpha-1}-\left(\log \frac{t_{2}}{s}\right)^{\alpha-1} \frac{d s}{s}+\frac{\rho}{\Gamma(\alpha)} \int_{t_{1}}^{t_{2}}\left(\log \frac{t_{2}}{s}\right)^{\alpha-1} \frac{d s}{s} \\
\leq & \frac{\rho}{\Gamma(\alpha+1)}\left(\left(\log \frac{t_{1}}{)^{\alpha}}+\left(\log \frac{t_{2}}{t_{1}}\right)^{\alpha}-\left(\log t_{2}\right)^{\alpha}+\left(\log \frac{t_{2}}{t_{1}}\right)^{\alpha}\right)\right. \\
\leq & \frac{2 \rho}{\Gamma(\alpha+1)}\left(\log \frac{t_{2}}{t_{1}}\right)^{\alpha} .
\end{aligned}
$$


As $t_{1} \rightarrow t_{2}$ the right-hand side of the previous inequality is independent of $x$ and tends to zero. Thus that $\mathcal{A}\left(B_{\eta}\right)$ is equicontinuous. So, the compactness of $\mathcal{A}$ follows by Ascoli Arzela's theorem.

Now for any $x \in[a, b] \subset \mathbb{R}^{+}$, we define respectively the upper and lower control functions as follows

$$
H(t, x)=\sup _{a \leq y \leq x} f(t, y), h(t, x)=\inf _{x \leq y \leq b} f(t, y) .
$$

It is clear that these functions are nondecreasing on $[a, b]$.

Definition 10. Let $\bar{x}, \underline{x} \in \mathcal{E}, a \leq \underline{x} \leq \bar{x} \leq b$, satisfying

$$
\bar{x}(t) \geq \frac{1}{\Gamma(\alpha)} \int_{1}^{t}\left(\log \frac{t}{s}\right)^{\alpha-1} H(s, \bar{x}(s)) \frac{d s}{s}+\lambda \int_{1}^{e} \bar{x}(s) d s+d, \quad 1 \leq t \leq e,
$$

and

$$
\underline{x}(t) \leq \frac{1}{\Gamma(\alpha)} \int_{1}^{t}\left(\log \frac{t}{s}\right)^{\alpha-1} h(s, \underline{x}(s)) \frac{d s}{s}+\lambda \int_{1}^{e} \underline{x}(s) d s+d, \quad 1 \leq t \leq e .
$$

Then the functions $\bar{x}$ and $\underline{x}$ are called a pair of upper and lower solutions for the equation (1).

Theorem 11. Assume that $f:[1, e] \times[0, \infty) \rightarrow[0, \infty)$ is continuous, and $\bar{x}$ and $\underline{x}$ are respectively upper and lower solutions of (1), then the problem (1) has at least one positive solution.

Proof. Let

$$
\mathcal{K}=\{x \in \mathcal{E}, \underline{x}(t) \leq x(t) \leq \bar{x}(t), t \in[1, e]\} .
$$

As $\mathcal{K} \subset \mathcal{E}$ and $\mathcal{K}$ is a nonempty bounded, closed and convex subset. By Lemma 9, $\mathcal{A}: \mathcal{K} \rightarrow \mathcal{E}$ is completely continuous. Next, we show that if $x \in \mathcal{K}$, we have $\mathcal{A} x \in \mathcal{K}$. For any $x \in \mathcal{K}$, we have $\underline{x} \leq x \leq \bar{x}$, then

$$
\begin{aligned}
(\mathcal{A} x)(t) & =\frac{1}{\Gamma(\alpha)} \int_{1}^{t}\left(\log \frac{t}{s}\right)^{\alpha-1} f(s, x(s)) \frac{d s}{s}+\lambda \int_{1}^{e} x(s) d s+d \\
& \leq \frac{1}{\Gamma(\alpha)} \int_{1}^{t}\left(\log \frac{t}{s}\right)^{\alpha-1} H(s, \bar{x}(s)) \frac{d s}{s}+\lambda \int_{1}^{e} \bar{x}(s) d s+d \\
& \leq \bar{x}(t)
\end{aligned}
$$

and

$$
\begin{aligned}
(\mathcal{A} x)(t) & =\frac{1}{\Gamma(\alpha)} \int_{1}^{t}\left(\log \frac{t}{s}\right)^{\alpha-1} f(s, x(s)) \frac{d s}{s}+\lambda \int_{1}^{e} x(s) d s+d \\
& \geq \frac{1}{\Gamma(\alpha)} \int_{1}^{t}\left(\log \frac{t}{s}\right)^{\alpha-1} h(s, \underline{x}(s)) \frac{d s}{s}+\lambda \int_{1}^{e} \underline{x}(s) d s+d \\
& \geq \underline{x}(t) .
\end{aligned}
$$

Thus, from (4) and (5), we obtain that $\mathcal{A} x \in \mathcal{K}$. We now see that all the conditions of the Schauder fixed point theorem are satisfied. Thus there exists a fixed point $x$ in $\mathcal{K}$. Therefore, the problem (1) has at least one positive solution $x$ in $\mathcal{K}$.

Corollary 12. Assume that $\lambda=0$ and $f:[1, e] \times[0, \infty) \rightarrow[0, \infty)$ is continuous, and there exist $\lambda_{1}, \lambda_{2}>0$ such that

$$
\lambda_{1} \leq f(t, x) \leq \lambda_{2},(t, x) \in[1, e] \times[0,+\infty)
$$

Then the problem (1) has at least one positive solution $x \in \mathcal{E}$, moreover

$$
x(t) \geq \lambda_{1} \frac{(\log t)^{\alpha}}{\Gamma(\alpha+1)}+d,
$$


and

$$
x(t) \leq \lambda_{2} \frac{(\log t)^{\alpha}}{\Gamma(\alpha+1)}+d .
$$

Proof. From (6) and the definition of control functions, we have

$$
\lambda_{1} \leq h(t, x) \leq H(t, x) \leq \lambda_{2} .
$$

Now, let

$$
\mathfrak{D}_{1}^{\alpha} \bar{x}(t)=\lambda_{2}, \bar{x}(1)=d .
$$

The above equation (10) has a positive solution

$$
\bar{x}(t)=\frac{\lambda_{2}}{\Gamma(\alpha)} \int_{1}^{t}\left(\log \frac{t}{s}\right)^{\alpha-1} \frac{d s}{s}+d=\lambda_{2} \frac{(\log t)^{\alpha}}{\Gamma(\alpha+1)}+d .
$$

Taking into account (9), we have

$$
\bar{x}(t)=\frac{\lambda_{2}}{\Gamma(\alpha)} \int_{1}^{t}\left(\log \frac{t}{s}\right)^{\alpha-1} \frac{d s}{s}+d \geq \frac{1}{\Gamma(\alpha)} \int_{1}^{t}\left(\log \frac{t}{s}\right)^{\alpha-1} H(s, \bar{x}(s)) \frac{d s}{s}+d .
$$

It is clear that $\bar{x}$ is the upper solution of (1). Now, let

$$
\mathfrak{D}_{1}^{\alpha} \underline{x}(t)=\lambda_{1}, \underline{x}(1)=d .
$$

which has also a positive solution

$$
\underline{x}(t)=\frac{\lambda_{1}}{\Gamma(\alpha)} \int_{1}^{t}\left(\log \frac{t}{s}\right)^{\alpha-1} \frac{d s}{s}+d=\lambda_{1} \frac{(\log t)^{\alpha}}{\Gamma(\alpha+1)}+d .
$$

By (9) and the same way that we used to search the upper solution, we conclude also that $\underline{x}$ is the lower solution of (1). Therefore, from Theorem 11, we conclude that the problem (1) has at least one positive solution $x \in \mathcal{E}$ which verifies the inequalities (7) and (8).

\section{Uniqueness of positive solutions}

In this section, we shall prove the uniqueness of positive solutions using the contraction mapping principle.

Theorem 13. Assume that $f:[1, e] \times[0, \infty) \rightarrow[0, \infty)$ is continuous and there exists $L>0$ such that

$$
\left|f\left(t, x_{1}\right)-f\left(t, x_{2}\right)\right| \leq L\left|x_{1}-x_{2}\right| \text { for any } t \in[1, e], x_{1}, x_{2} \in[0, \infty),
$$

with

$$
\frac{L}{\Gamma(\alpha+1)}+\lambda(e-1)<1
$$

Then the problem (1) has a unique positive solution $x \in \mathcal{K}$.

Proof. From Theorem 11, it follows that (1) has at least one positive solution in $\mathcal{K}$. Hence, we need only to prove that the operator $\mathcal{A}$ defined in (3) is a contraction on $\mathcal{K}$. In fact, since for any $x_{1}, x_{2} \in \mathcal{K}$, (11) and (12) are verified, then we have

$$
\begin{aligned}
\left|\left(\mathcal{A} x_{1}\right)(t)-\left(\mathcal{A} x_{2}\right)(t)\right| & \leq \frac{1}{\Gamma(\alpha)} \int_{1}^{t}\left(\log \frac{t}{s}\right)^{\alpha-1}\left|f\left(s, x_{1}(s)\right)-f\left(s, x_{2}(s)\right)\right| \frac{d s}{s}+\lambda \int_{1}^{e}\left|x_{1}(s)-x_{2}(s)\right| d s \\
& \leq \frac{(\log t)^{\alpha}}{\Gamma(\alpha+1)} L\left\|x_{1}-x_{2}\right\|+\lambda(e-1)\left\|x_{1}-x_{2}\right\| \leq\left(\frac{L}{\Gamma(\alpha+1)}+\lambda(e-1)\right)\left\|x_{1}-x_{2}\right\| .
\end{aligned}
$$


Thus,

$$
\left\|\mathcal{A} x_{1}-\mathcal{A} x_{2}\right\| \leq\left(\frac{L}{\Gamma(\alpha+1)}+\lambda(e-1)\right)\left\|x_{1}-x_{2}\right\| .
$$

Hence, the operator $\mathcal{A}$ is a contraction mapping by (12). Therefore, by the contraction mapping principle, we conclude that the problem (1) has a unique positive solution $x \in \mathcal{K}$.

Now, we give an example to illustrate our results.

Example 1. We consider the following nonlinear fractional differential equation

$$
\left\{\begin{array}{l}
\mathfrak{D}_{1}^{\frac{2}{5}} x(t)=\frac{1}{3+t}\left(\frac{t-1}{x(t)+1}+2\right), 1<t \leq e, \\
x(1)=1
\end{array}\right.
$$

where $\alpha=2 / 5, \lambda=0, d=1$ and $f(t, x)=\frac{1}{3+t}\left(\frac{t-1}{x+1}+2\right)$. Since $f$ is continuous and

$$
\frac{2}{3+e} \leq f(t, x) \leq \frac{1}{2}
$$

for $(t, x) \in[1, e] \times[0, \infty)$, then by Corollary $12,(13)$ has a positive solution which verifies $\underline{x}(t) \leq x(t) \leq \bar{x}(t)$ where

$$
\bar{x}(t)=\frac{1}{2} \frac{(\log t)^{2 / 5}}{\Gamma(7 / 5)}+1 \text { and } \underline{x}(t)=\frac{2}{3+e} \frac{(\log t)^{2 / 5}}{\Gamma(7 / 5)}+1,
$$

are respectively the upper and lower solutions of (13). Also, we have

$$
\frac{L}{\Gamma(\alpha+1)} \simeq 0.339<1
$$

then by Theorem 13, (13) has a unique positive solution which is bounded by $\underline{x}$ and $\bar{x}$.

\section{Conclusion}

In this paper, the existence and uniqueness of positive solutions for nonlinear Caputo-Hadamard fractional differential equations with integral boundary conditions were obtained using the Schauder fixed point theorem and the Banach contraction mapping principle. The obtained results extend some known results in the literature. An example is introduced to illustrate the main results of this paper.

Acknowledgments: The authors would like to thank the anonymous referee for his/her valuable comments and good advice.

Author Contributions: All authors contributed equally to the writing of this paper. All authors read and approved the final manuscript.

Conflicts of Interest: "The authors declare no conflict of interest."

\section{References}

[1] Abbas, S. (2011). Existence of solutions to fractional order ordinary and delay differential equations and applications. Electronic Journal of Differential Equations (EJDE), 2011(9), 1-11.

[2] Abdo. M. A., Wahash. H. A., \& Panchat, S. K. (2018). Positive solutions of a fractional differential equation with integral boundary conditions. Journal of Applied Mathematics and Computational Mechanics 17(3) (2018), 5-15.

[3] Agarwal, R. P., Zhou, Y., \& He, Y. (2010). Existence of fractional neutral functional differential equations. Computers E Mathematics with Applications, 59(3), 1095-1100.

[4] Ahmad, B., \& Ntouyas, S. K. (2017). Existence and uniqueness of solutions for Caputo-Hadamard sequential fractional order neutral functional differential equations. Electronic Journal of Differential Equations, 2017(36), 1-11.

[5] Boulares, H., Ardjouni, A., \& Laskri, Y. (2017). Positive solutions for nonlinear fractional differential equations. Positivity, 21(3), 1201-1212.

[6] Boulares, H., Ardjouni, A., \& Laskri, Y. (2016). Stability in delay nonlinear fractional differential equations. Rendiconti del Circolo Matematico di Palermo Series 2, 65(2), 243-253. 
[7] Chidouh, A., Guezane-Lakoud, A., \& Bebbouchi, R. (2016). Positive solutions of the fractional relaxation equation using lower and upper solutions. Vietnam Journal of Mathematics, 44(4), 739-748.

[8] Ge, F., \& Kou, C. (2015). Stability analysis by Krasnoselskii's fixed point theorem for nonlinear fractional differential equations. Applied Mathematics and Computation, 257, 308-316.

[9] Ge, F., \& Kou, C. (2015). Asymptotic stability of solutions of nonlinear fractional differential equations of order $1<\alpha<2$. Journal of Shanghai Normal University (Natural Sciences), 44(3) (2015), 284-290.

[10] Kilbas, A. A. A., Srivastava, H. M., \& Trujillo, J. J. (2006). Theory and applications of fractional differential equations (Vol. 204). Elsevier Science Limited.

[11] Kou, C., Zhou, H., \& Yan, Y. (2011). Existence of solutions of initial value problems for nonlinear fractional differential equations on the half-axis. Nonlinear Analysis: Theory, Methods \& Applications, 74(17), 5975-5986.

[12] Lakshmikantham, V., \& Vatsala, A. S. (2008). Basic theory of fractional differential equations. Nonlinear Analysis: Theory, Methods \& Applications, 69(8), 2677-2682.

[13] Nan, L. I., \& Changyou, W. A. N. G. (2013). New existence results of positive solution for a class of nonlinear fractional differential equations. Acta Mathematica Scientia, 33(3), 847-854.

[14] Podlubny, I. (1998). Fractional differential equations: an introduction to fractional derivatives, fractional differential equations, to methods of their solution and some of their applications (Vol. 198). Elsevier.

[15] Smart, D. R. (1980). Fixed point theorems (Vol. 66). CUP Archive.

[16] Zhang, S. (2000). The existence of a positive solution for a nonlinear fractional differential equation. Journal of Mathematical Analysis and Applications, 252(2), 804-812. 